\title{
Biometria, caracterização física e rendimento lipídico do fruto de Licania rigida Benth adquiridos no município de Pombal-PB
}

\author{
Biometrics, physical characteristics and yield fruit of lipid Licania rigid Benth \\ purchased in the municipality of Pombal-PB
}

\author{
Thalita Sévia Soares de Almeida ${ }^{1}$, Alfredina dos Santos Araújo ${ }^{2}$, Gilcean Silva Alves ${ }^{3}$, Ana Emília Formiga Marque ${ }^{4}$, Tiago da \\ Nóbrega Albuquerque 5
}

\begin{abstract}
Resumo: Licânia rigida Benth, popularmente oiticica, é uma vegetação típica no sertão nordestino. Destaca-se pelo alto teor de óleos em seus frutos e boa produtividade mesmo em épocas de seca, sendo capaz de promover desenvolvimento social e econômico para a região, pela utilização industrial de tintas, vernizes, sabão, lonas e esmaltes finos. Portanto, objetiva-se no presente estudo determinar a biometria, parâmetros físicos e rendimento lipídico do fruto da oiticica. Os frutos foram colhidos, selecionados, sanitizados. Em seguida, utilizou-se um paquímetro para a realização da biometria dos frutos e então sofreram separação em exocarpo-mesocarpo, endocarpo e amêndoas. As amostras foram trituradas e armazenadas ate procedidas as análises. Para a determinação do pH e condutividade, utilizou-se cerca de $5 \mathrm{~g}$ de cada amostra, acrescidos de $50 \mathrm{~mL}$ de água destilada e homogeneização, por conseguintemente foi utilizado o peagâmetro digital e condutivímetro, respectivamente. Para a acidez total titulável (ATT), utilizou-se as amostras anteriores e titulou-se com $\mathrm{NaOH} 0,1 \mathrm{~N}$, usando fenolftaleína como indicador, até as amostras alcançarem pH de 8,1. Procedeu-se a determinação do teor lipídico pelo método de Sohlext, com 2 a $5 \mathrm{~g}$ das amostras e hexano como solvente. Os frutos apresentaram peso médio de $9 \mathrm{~g}$. A variação do $\mathrm{pH}$ para as diferentes amostra foi irrelevante, enquanto o exocarpo-mesocarpo apresentou maior condutividade, com $308,6 \mathrm{mScm}^{-1}$, e o endocarpo foi evidenciado por apresentar maior ATT, com 16,8\%. Obteve-se na amêndoa maior percentual lipídico, com rendimento médio de 43,0\%. Diante da importância e alta rentabilidade lipídica do fruto, faz-se necessário desenvolvimento de metodologias para utilização dessa matéria-prima.
\end{abstract}

Palavras-chaves: Oiticica. Parâmetros físicos. Lipídios.

\begin{abstract}
Licania rigid Benth, popularly oiticica, is typical vegetation in the northeastern hinterland. It contains high oil content in its fruits and good productivity even in times of drought; promote social and economic development for the region, the industrial use of paints, varnishes, soap, tarpaulins and thin glazes. Therefore, this study aims determine biometrics, physical parameters and lipid yield the fruit of the myrtle. The fruits were harvested, sorted, sanitized. Then, a caliper was used to carry out the biometrics fruit and then underwent separation exocarp-mesocarp and endocarp almonds. The samples were ground and stored until analysis proceeded. For the determination of $\mathrm{pH}$ and conductivity was used about $5 \mathrm{~g}$ of each sample plus $50 \mathrm{ml}$ of distilled water and homogenizing for then digital $\mathrm{pH}$ meter was used and conductivity, respectively. For titratable acidity (TA) was used earlier samples, and titrated with $0.1 \mathrm{~N} \mathrm{NaOH}$, using phenolphthalein as an indicator until the samples reach a $\mathrm{pH}$ of 8.1 . The procedure was the determination of the lipid content by Sohlext method, of 2 to $5 \mathrm{~g}$ of sample and hexane as solvent. The fruits showed an average weight of $9 \mathrm{~g}$. The $\mathrm{pH}$ changes for the various samples were irrelevant, while the exocarp-mesocarp showed higher conductivity, with 308.6 MSCM-1, and the endocarp was evidenced by higher ATT present with $16.8 \%$. It was obtained in the almond lipid largest percentage, with average yield of $43.0 \%$. Given the importance and high lipid profitability of the fruit, it is necessary to develop methodologies for use of this raw material.
\end{abstract}

Key words: Oiticica. Physical parameters. Lipids.

\footnotetext{
*Autor para correspondência

Recebido para publicação em 20/11/2015; aprovado em 10/12/2015

${ }^{1}$ Mestre em Sistemas Agroindustriais, Universidade Federal de Campina Grande, Pombal - PB, E-mail: thalitasevia22@ gmailo.com

${ }^{2}$ Doutora em Engenharia de Processos, Universidade Federal de Campina Grande, Campina Grande - PB, E-mail: alfredinad@hotmail.com

${ }^{3}$ Doutor em Agronomia, Universidade Federal da Paraíba, João Pessoa - PB E-mail: biopb@ hotmail.com

${ }^{4}$ Mestranda em Ciências Naturais, Universidade Federal de Campina Grande, Cuité - PB, E-mail: anaemiliaformiga@hotmail.com

${ }^{5}$ Graduando em Engenharia de Alimentos, Universidade Federal de Campina Grande, Pombal - PB, E-mail: tiagofernandes_pb@ hotmail.com
} 


\section{INTRODUÇÃO}

A Licânia rigida Benth, vegetação conhecida regionalmente como oiticica, pertence à família Crysobalanaceae, estabelecendo típica de matas ciliares da caatinga, cresce em aluviões profundos das bacias hidrográficas dos rios e riachos que se encontram nas regiões do Piauí, Ceará, Rio Grande do Norte e Paraíba, principalmente no Sertão (SILVA FILHO, 2010).

As oiticicas atingem aproximadamente 20 metros de altura e o seu tronco grosso ramifica-se a pouca distância do solo. Japiassú, Lopes e Dantas (2008) afirmam que o seu acentuado xerofilismo caracteriza-se pela perenidade de suas folhas grossas e ásperas, revestidas de cutícula espessa que protege a sua superfície contra a evaporação, além de um sistema radicular profundo, cobrindo maior área do solo e permitindo bom suprimento aos seus mecanismos de absorção e transporte de água. As flores são creme-amareladas, dispostas em espigas racemosas, em longos cachos, chegando a medir três milímetros de diâmetro (SANTIAGO, 2005).

A frutificação varia de tamanho e composição conforme a espécie da planta. Estudos realizados por Melo et al. (2006) apontaram os seus frutos apresentaram-se drupáceo, fusiforme ou ovalado, medindo de 4 a $6 \mathrm{~cm}$ de comprimento, com cerca de dois centímetros de diâmetro, peso médio de 4 a 7 gramas e coloração castanha.

O fruto é formado por uma cápsula, composta de três camadas, tais quais exocarpo, cuja casca é verde quando maduro e torna-se amarelo-escuro quando amadurece e seca; mesocarpo, porção mediana, sucosa por ser rica em água, e endocarpo apresentando-se como uma massa amarelada, rala e fibrosa, ambas com função de revestir e proteger a semente, esta caracterizada por uma coloração marrom semelhante à madeira, composta por uma casca revestindo a amêndoa.

Segundo Bayma (1957), o endocarpo pode ser utilizado como aditivo em ração para o gado, mas é da amêndoa que se extrai o maior teor óleo, utilizado em larga escala pelas indústrias produtora de tintas automotivas, de impressoras, vernizes, esmaltes finos e lonas devido ao seu alto poder secativo e, ainda, na fabricação de sabão, sendo um produto secundário de reações químicas procedidas com o óleo, a partir do processo de saponificação.

Desde 1843 já se pensava na extração e exploração do óleo da oiticica para fins industriais, mas em virtude dos poucos conhecimento e estudos a respeito das características do óleo e a falta de tecnologias de extração eficientes, grandes projetos fracassaram ao longo do tempo. Foi somente por volta de 1930, quando estudos desenvolvidos por químicos, conseguiram desenvolver um tratamento do óleo e preparação de tintas para madeira, desde então a cultura da oiticica alavancou com a criação de grandes indústrias e comercialização do óleo (PINTO, 1963).

Os nordestinos destas regiões viveram tempos de apogeu, visto que a produção de óleo era de fundamental importância na manutenção da renda de famílias que subsistiam na região da caatinga, já que a colheita era realizada entre os meses de dezembro e fevereiro, período tradicional de total escassez de recursos financeiros pela agricultura familiar. Os produtores do município de Pombal e vizinhanças viveram um período de progresso em decorrência da forte geração de emprego e renda proveniente da exploração da oiticica, uma vez que foi instalada no
Município de Pombal - PB uma das 17 filiais da maior indústria extratora e compradora de óleos do país, denominada de Brasil Oiticica (QUEIROGA, 2013).

Segundo Duque (2004) ao longo dos anos foi havendo quedas no mercado e exportação do óleo de oiticica em virtude da utilização de outros, tais como o óleo de perila, tungue e linhaça. Além da formação de reações indesejáveis ocorrentes quando o óleo de oiticica era exposto à atmosfera, pouco foi o investimento afim do melhoramento, otimização e desenvolvimento de novas técnicas de exploração, portanto conduziu-se ao fechamento das grandes indústrias, tornando a prática quase completamente abandonada, apresentando reflexos negativos em toda a cadeia produtiva desta atividade (QUEIROGA, 2013).

Atualmente, a utilização do óleo da oiticica tem sido evidenciada, tanto por parte da indústria química fina na produção de biolubrificantes e biopolímeros como indústria farmacêutica e de cosméticos, além de provocar grandes discursões quanto à capacidade de formulação de um novo biodiesel, como um biocombustível renovável (OLIVEIRA, et al., 2012; MORAIS, 2015).

Para tanto, faz-se necessário o devido conhecimento quanto à caracterização física e química do fruto, bem como a investigação e análise de seus potenciais. Portanto, diante do exposto, objetivou-se no presente estudo determinar parâmetros físicos e rendimento lipídico, para as diferentes partes do fruto da oiticica.

\section{MATERIAL E MÉTODOS}

Para o presente estudo foram utilizadas amostras do fruto da oiticica adquiridas em um vegetal pertencente ao localizado no Município de Pombal - Paraíba, zona urbana, localizado a três $\mathrm{km}$ do centro do município.

Os frutos maduros foram coletados e armazenados em sacos plásticos e estéreos, identificados e acondicionados em caixas isotérmicas para transporte, logo encaminhados ao Laboratório Centro Vocacional e Tecnológico (CVT) pertencente à Universidade Federal de Campina Grande (UFCG) campus Pombal - Paraíba, onde foram submetidas às análises. No laboratório os frutos foram submetidos à sanitização e seleção das amostras, com objetivo de selecionar frutos considerados adequados para análise, de boa aparência, garantindo a qualidade do produto final.

Procedeu-se a biometria de 50 frutos, utilizando-se de uma régua de paquímetro, no qual foi possível determinar a largura e comprimento do fruto. Obteve-se o peso médio dos frutos em balança analítica.

Para a obtenção das amostras em estudo, o fruto sofreu separação em exocarpo-mesocarpo, no qual foi realizado o descascamento (retirada das cascas do fruto) de forma manual, com auxílio de facas, endocarpo e sementes (Figura 1). Estas sofreram secagem em estufa de circulação de ar forçada, marca Sterilifer e modelo CR/100, à temperatura de $105^{\circ} \mathrm{C}$, por período de 24 horas, por conseguintemente procedeu-se a trituração em liquidificador industrial, para a formação de grãos. Então, foram procedidas análises físicas e teor lipídico para as amostras processadas. Os testes foram realizados em triplicata e seguidos métodos descritos pelo Instituto Adolfo Lutz (IAL) (2008). 
Figura 1. (A) Amostras do endocarpo obtidas após processo de secagem; (B) Amostras de sementes obtidas após processo de secagem.

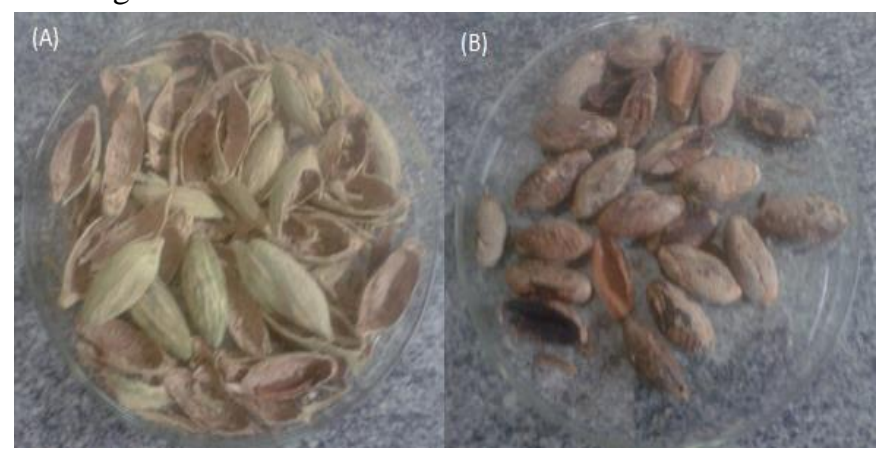

\section{Potencial Hidrogeniônico (pH)}

Para a determinação do $\mathrm{pH}$, foram pesados cerca de $5 \mathrm{~g}$ de cada amostra e transferidos para béqueres, nos quais foram acrescidos de $50 \mathrm{~mL}$ de água destilada para homogeneização. $\mathrm{O} \mathrm{pH}$ foi determinado através do método potenciométrico, com peagâmetro de bancada da marca Lucadema e modelo mPA, previamente calibrado com solução tampão de $\mathrm{pH} 4,00$ e 7,00 .

\section{Condutividade Elétrica}

A condutividade elétrica foi determinada por um condutivímetro de bancada para soluções aquosas Lucadema e modelo mCA 150, com faixa de leitura de 0 a 20.000 $\mathrm{mScm}^{1}$ (4 escalas), célula de condutividade tipo caneta e constante de eletrodo 1,0 . Os cálculos realizados consideraram os pesos das amostras e valor expresso no condutivímetro. Os resultados foram expressos em $\mathrm{mScm}^{1}$.

\section{Acidez Total Titulável (ATT)}

Após determinação do pH, as amostras sofreram titulação com solução de hidróxido de sódio $(\mathrm{NaOH}) 0,1 \mathrm{~N}$ e, com o auxílio do peagâmetro de bancada, foi possível ser determinado o volume titulável, para isso, as amostras tiveram que alcançar $\mathrm{pH}$ de 8,1. Foi utilizado ainda fenolftaleína como indicador. Os cálculos realizados consideraram o peso das amostras utilizadas, volume de $\mathrm{NaOH} 0,1 \mathrm{~N}$ consumido. Os resultados foram expressos em porcentagem.

\section{Lipídios}

Para a determinação de lipídios foi medido $2 \mathrm{a} 5 \mathrm{~g}$ de cada amostra e acrescentou-se hexano como solvente extrator em aparelho de Sohlext. O sistema sofreu aquecimento por aproximadamente 6 horas e em seguida os balões volumétricos de fundo chato foram submetidos a secagem em estufa de circulação de ar à $105^{\circ} \mathrm{C}$, por período de uma hora, assim permitiu-se para retirada do solvente excedente e a gordura extraída pôde ser quantificada. Os cálculos realizados consideraram o peso das amostras utilizadas, peso dos balões antes e depois do processo de extração. Os resultados foram expressos em porcentagem.

\section{RESULTADOS E DISCUSSÃO}

A partir da biometria realizada obtiveram-se os valores dispostos na Tabela 1.

Tabela 1. Avaliação biométrica do fruto da oiticica.

\begin{tabular}{lcccc}
\hline \multirow{2}{*}{ BIOMETRIA } & \multicolumn{4}{c}{ RESULTADOS } \\
\cline { 2 - 5 } & MÉDIA & MÁX. & MÍN. & DP (\%) \\
\hline $\begin{array}{l}\text { Comprimento } \\
\text { (cm) }\end{array}$ & 3,94 & 4,70 & 2,00 & 0,34 \\
Largura (cm) & 1,76 & 2,30 & 1,10 & 0,19 \\
Peso (g) & 9,07 & 14,90 & 2,45 & 1,96 \\
\hline
\end{tabular}

Dados: obtidos em estudo.

O fruto da oiticiqueira apresentou comprimento médio de $3,95 \mathrm{~cm}$ e $1,76 \mathrm{~cm}$ de largura, enquanto o peso médio foi de aproximadamente $9 \mathrm{~g}$. Estudos realizados por Melo et. al. (2006) apontou que o fruto pode pesar cerca de 4 a $7 \mathrm{~g}$, apresentando variação significativa enquanto ao determinado em estudo, capaz de atingir 14,9g/fruto.

Ao analisar os dados obtidos foi percebido que não houve variação relevante quanto ao $\mathrm{pH}$ entre as amostra do exocarpo-mesocarpo, endocarpo e amêndoa. A condutividade elétrica, a fim de prevê a padronização do teor de água inicial de cada amostra apresentou-se relativamente baixa para as três tipos de amostras, no entanto foi determinado maior valor para o exocarpo-mesocarpo, porções mais externas do fruto, sendo a amêndoa a apresentar menor quantidade de água em sua constituição, provavelmente menor teor eletrolítica. Quanto a ATT, foi para a amêndoa o maior teor percentual, com aproximadamente $16,8 \%$ (Tabela 2).

Tabela 2. Análises físicas e teor lipídico das diferentes partes do fruto da oiticica.

\begin{tabular}{lccc}
\hline \multirow{2}{*}{ ANÁLISES } & & RESULTADOS & Amêndoa \\
\cline { 2 - 4 } Potencial Hidrogeniônico & Exocarpo-Mesocarpo & Endocarpo & 5,4 \\
Condutividade $\left(\mathrm{mScm}^{-1}\right)$ & 5,4 & 5,6 & 0,9 \\
Acidez Total Titulável (\%) & 308,6 & 126,0 & 7,3 \\
Lipídios $(\%)$ & 8,2 & 16,8 & 43,0 \\
\hline
\end{tabular}

Dados: obtidos em estudo. 
O rendimento lipídico demonstrou-se elevado para as amostras de exocarpo-mesocarpo e endocarpo, com 12,8 e 9,5\% respectivamente, no entanto, como previsto, o maior percentual apresentado foi para as amostras de amêndoas, com $43,0 \%$.

Em análises realizadas por Almeida (2015) determinouse o teor lipídico em amostras de exocarpo-mesocarpo, com $17,9 \%$, mostrando-se distinto do disposto em o presente trabalho, enquanto o endocarpo dispôs de 9,8\%.

Estudos realizados por Pinto (1963) apresentou 60 a $63 \%$ de teor lipídico em amêndoas de oiticiqueira, enquanto que trabalhos realizados por Oliveira et al. (2012) apontaram $65 \%$ de rendimento do óleo, assim pode-se concluir que não houve variação na concentração de lipídicos em função do tempo e localização da coleta dos frutos, no entanto, apesar do alto teor lipídico encontrado em amêndoas em o presente estudo, o valor determinado não condiz com dados encontrados em literaturas supracitadas, uma vez que corresponde à aproximadamente $67 \%$ de gordura em relação aos estudos semelhantes. Acredita-se que tal variação pôde ser dada devido à utilização de diferentes metodologias quanto ao processo de secagem das amostras, em se comparando com o estudo realizado por Oliveira et. al. (2012).

\section{CONCLUSÃO}

É de fundamental importância a realização das análises para determinação de demais componentes do fruto, uma vez que, a utilização do produto pode ser ampliada de acordo com suas propriedades e constituintes químicos.

Diante da utilização do óleo da oiticica e valor quanto à possível contribuição social e econômica para a região do alto sertão, é percebido a necessidade do desenvolvimento, bem como adoção de técnicas adequadas para o processo de extração do óleo e demais análises adotadas, portanto sugerese o contínuo estudo deste vegetal para que haja o melhor aproveitamento do fruto, em virtude da alta rentabilidade lipídica.

\section{REFERÊNCIAS BIBLIOGRÁFICAS}

ALMEIDA, T. S. S. DE. Caracterização físico-química do fruto e susceptibilidade antimicrobiana do óleo de Licania rigida Benth (Oiticica). Dissertação (Mestrado em Sistemas Agroindustriais) - Programa de Pós-Graduação em Sistemas Agroindustriais, Universidade Federal de Campina Grande, 2015.

BAYMA, C. Oiticica. Ministério da Agricultura - SAI. Rio de Janeiro. (Produtos Rurais 1), 1957. 53p.

DUQUE, G. O Nordeste e as lavouras xerófilas. 4. ed. Fortaleza: Banco do Nordeste do Brasil, 2004. 330p.

INSTITUTO ADOLFO LUTZ. Métodos físico-químicos para análises de alimentos. (Coor.) ZENEBON O.; PAUCUET, N. S.; TIGLEA P. São Paulo: Instituto Adolfo Lutz, 2008. 1020p.
JAPIASSÚ A.; LOPES, K. P.; DANTAS, J. G. Caracterização fenológica da oiticica (LICANIA RIGIDA BENTH). 59 Congresso Nacional de Botânica. 2008.

MELO, J. C.; TEIXEIRA, J. C.; BRITO, J. Z. ; PACHECO, J. G. A.; STRAGEVITCH, L. Produção de biodiesel de óleo de oiticica. In: Congresso Brasileiro de Tecnologia de Biodiesel, Brasília, DF. Anais... Brasília, DF: MCT/ABIPTI, 2006. p.165-167.

MORAIS, LEANDRO VINICIUS FERNANDES. Atividade antimicrobiana e antioxidante de Licania rigida e Turnera ulmifolia. Dissertação (Mestrado Ciências Farmacêuticas) - Programa de Pós-Graduação em Ciências Farmacêuticas do CCS, Universidade Federal do Rio Grande do Norte, 2015.

OLIVEIRA, F. A. G. de; PINTO, V. de L.; DI SOUZA L.; DINIZ, J. C.; SANTOS, A. G. D. dos; VIANNA, F. A. Síntese, caracterização e avaliação de biodiesel de óleo de oiticica (Licania Rigida Benth) e isolalamento do éster metílico do ácido licânico. Química: ciência, tecnologia e sociedade, v. 1, n. 1, 2012.

PINTO, G. P. Características físico-químicas e outras informações sobre as principais oleaginosas do Brasil. Recife: Instituto de Pesquisa e Experimentação Agropecuárias do Nordeste. p. 83, 1963. (Boletim Técnico, 18).

QUEIROGA, V. de P.; FREIRE, R. M. M.; ALMEIDA, C. MARINHO, D. R. de F.; MELO B. A. de. Composição química e mineral de amêndoas de oiticica em três tempos de armazenamento. Revista Verde, v.8, n.2, p.173-177, abr/jun, 2013.

SANTIAGO, G. P. AVALIAÇÃO DOS EFEITOS DE EXTRATOS AQUOSOS DE PLANTAS SOBRE A BIOLOGIA DA LAGARTA-DO-CARTUCHO, Spodoptera frugiperda (J. E. Smith, 1797) MANTIDA EM DIETA ARTIFICIAL. Dissertação (Mestrado em Agronomia) - Universidade Federal do Piauí. Teresina, 2005.

SILVA FILHO, J. P. da; SILVA, R. A,; SILVA, M. J. S. da. Potencial apícola para apis mellifera l. em área de caatinga no período da floração da oiticica (Licania rigida Benth). Revista Verde de Agroecologia e Desenvolvimento Sustentável, v.5, n. 1, 2010. 